\title{
Erratum to: Epidermoid Cyst of the Spleen
}

\author{
Yin-Yin Chen • Yi-Ming Shyr • Shin-E Wang
}

Received: 15 February 2013 / Accepted: 27 March 2013 /Published online: 3 December 2013

(C) 2013 The Society for Surgery of the Alimentary Tract

\section{Erratum to: Journal of Gastrointestinal Surgery}

\section{DOI 10.1007/s11605-012-2088-y}

Dear Editor of Journal of Gastrointestinal Surgery:

The article "Epidermoid Cyst of the Spleen" published in the following issue of Journal of Gastrointestinal Surgery: Volume 17, Issue 3 (2013), Page 555-561

The affiliated institute for each author which was stated in the galley proof shown below:

"From Departments of Surgery, Taipei Veterans General Hospital and National Yang Ming University, Taipei, Taiwan" was not seen in the published version of your journal.

An errata of the affiliated institute for each author should be corrected to be as follow:

"From Departments of Surgery, Taipei Veterans General Hospital and National Yang Ming University, Taipei, Taiwan"

The online version of the original article can be found at http://.dx.doi.org/ 10.1007/s11605-012-2088-y.

Y.-Y. Chen · Y.-M. Shyr · S.-E. Wang $(\bowtie)$

Departments of Surgery, Taipei Veterans General Hospital,

National Yang Ming University, Taipei, Taiwan

e-mail: sewang0408@gmail.com 